\title{
Effectiveness of Cognitive- Behavioral Therapy on Emotional Control and Signs of Suicide in Veterans with PTSD
}

\section{ART ICLE INF O}

\section{Article Type}

Original Research

\section{Authors}

Ramezani Sh.* $M A$,

Mohammadi N. ${ }^{1} M A$,

Sadri Damirchi E. ${ }^{1} P h D$,

Rahmani S. ${ }^{2} P h D$

How to cite this article
Ramezani Sh, Mohammadi N, Sa-
dri Damirchi E, Rahmani S. Effec-
tiveness of Cognitive- Behavioral
Therapy on Emotional Control
and Signs of Suicide in Veterans
with PTSD. Iranian Journal of W-
ar \& Public Health. 2018;10(1)
$: 1-7$.

*Education Department, Education \& Psychology Faculty, Kamyaran Branch, Payam-e-Noor University, Kurdestan, Iran

${ }^{1}$ Education Department, Education \& Psychology Faculty, University of Mohaghegh Ardabil, Ardabil, Iran ${ }^{2}$ Education Department, Education \& Psychology Faculty, University Campus Culture Shahid Modares, Sanandaj, Iran

\section{Correspondence}

Address: Sadegh Alley, Shariati Street, Kamyaran City, Kurdistan, Iran. Postal Code: 331657189 Phone: +98 (087) 35528280 Fax: +98 (087) 35524116 avinramezani@yahoo.com

\section{Article History}

Received: February 13, 2017

Accepted: September 26, 2017

ePublished: January 11, 2018

\section{A B S T R A C T}

Aims The existence of eight years of imposed war in Iran has caused a significant group of warriors and veterans to suffer from symptoms of post traumatic stress disorder (PTSD). PTSD is associated at least with signs of another psychiatric disorder. The purpose of this study was to investigate the effect of cognitive-behavioral therapy on emotional control and signs of suicide in veterans with PTSD.

Materials \& Methods In this semi-experimental study with pre-test and post-test design with a control group in 2017, 40 veterans with posttraumatic stress disorder hospitalized to Ghods Hospital of Sanandaj city were selected using available sampling. They were randomly assigned into two groups: experimental group (20 veterans) and control group (20 veterans). The data collection tools were Beck Scale for Suicidal Ideation (BSSI) and Emotional Control Questionnaire (ECQ). Patients in the experimental group received cognitive-behavioral therapy for 9 sessions. Data were analyzed by SPSS 16 software using univariate and multivariate covariance analysis.

Findings After modifying the pretest scores, there was a significant difference between experimental and control groups and cognitive-behavioral therapy increased emotional control $(F=25.40)$ and its subscales and reduced suicidal ideation $(F=38.19)$ and subscales in experimental group $(\mathrm{p}=0.001)$.

Conclusion Cognitive-behavioral therapy is effective in increasing emotional control and reducing suicidal signs in veterans with posttraumatic stress Keywords Cognitive-Behavioral Therapy; Emotional Disturbance; Suicidal Ideation; Veterans,
PTSD

\section{I T A T I O N L I N K S}

1] Diagnostic and statistical manual of mental ... [2] Predictive factors of chronic posttraumatic stress disorder 6 months after ... [3] I and post-traumatic stress ... [4] The study of effective factors on fmarital aladjusment ... [5] Prevalence of Post-Traumatic stress disorder and ... [6] Post-traumatic stress disorder in male chemical ... [7] in-depth look into PTSD-depression comorbidity ... [8] Examining the broader psychosocial effects of mass ... [9] Drinking motives and PTSD-related ... [10] The role of mindfulness as approach-based coping ... [11] PTSD, alcohol dependence, and conduct ... [12] An examination of sleep quality in ... [13] PTSD symptoms and suicide ideation: Testing the ... [14] Deficit in emotional intelligence underlying adolescent ... [15] The role of emotion regulation difficulties ... [16] Single-session emotion regulation skills training ... [17] Beliefs in negative mood regulation and ... [18] Psychiatric mental health ... [19] Suicidal ideation in adolescence an indicator ... [20] A global perspective in the epidemiology ... [21] Co-occurring aggression and suicide ... [22] Suicide risk among 1.3 Million veterans ... [23] Identification and management of suicide ... [24] Cognitive-behavioral intervention effects ... [25] The current state of cognitive therapy ... [26] Cognitive mechanisms of treatment in ... [27] Just showing up is not enough ... [28] The effects of cognitive behavioral therapy ... [29] Religious vs. conventional cognitive behavioral therapy ... [30] Physiological and behavioral indices of ... [31] Cognitive behavioral and pharmacological ... [32] Changes in the self during cognitive behavioral ... [33] A case study of individually delivered mindfulnessbased ... [34] The development of an integrated treatment ... [35] Effectiveness of brief VR treatment for PTSD ... [36] FMRI activation during executive ... [37] Timing matters: change depends on the stage ... [38] Cognitive behavioral therapy ... [39] Changes in threat-related cognitions ... [40] Assessing the reliability and validity ... [41] The relationship between emotional ... [42] The construction and preliminary ... [43] The construction and validation of a new scale ... [44] Comparison of the efficacy of cognitive behavior ... 


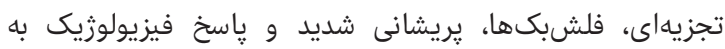

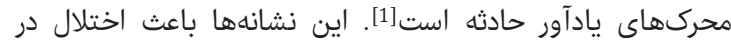

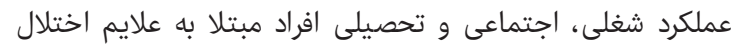

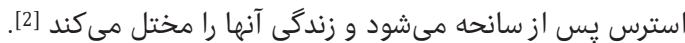

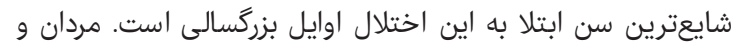

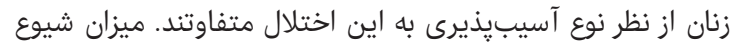

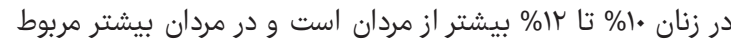

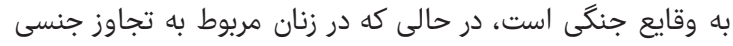

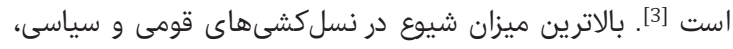

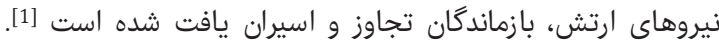

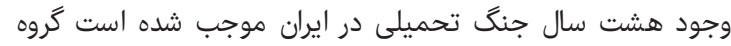

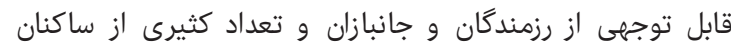

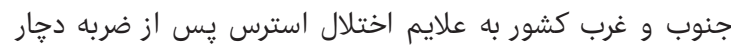

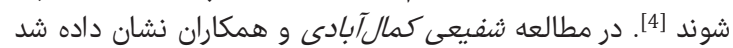

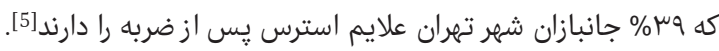

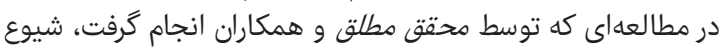

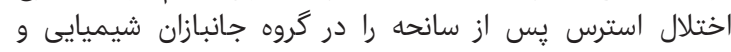

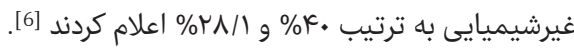

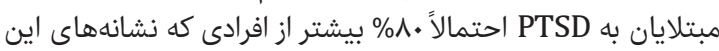

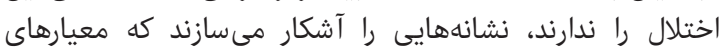

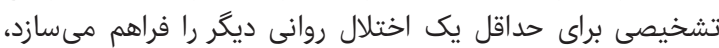

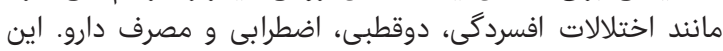

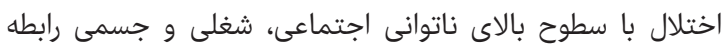

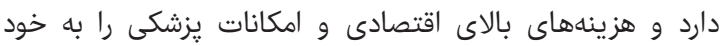

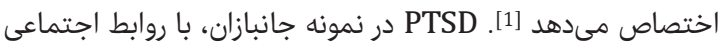

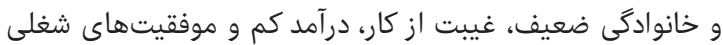

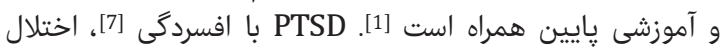

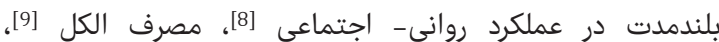

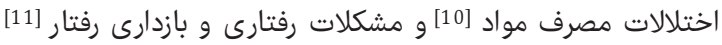

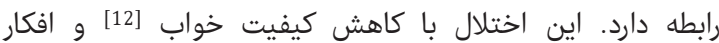
خودكشى [13] نيز همراه است.

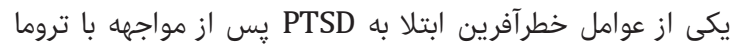

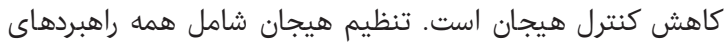

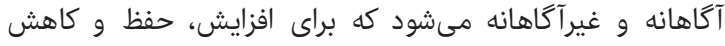

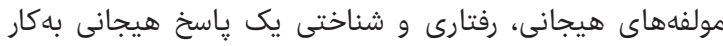

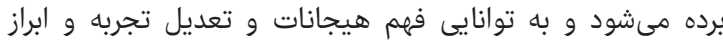

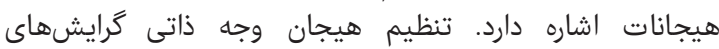

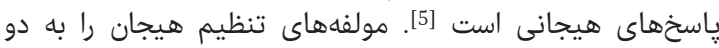

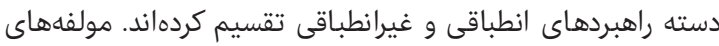

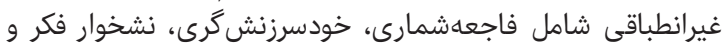

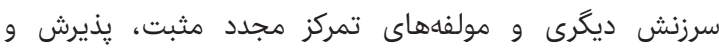

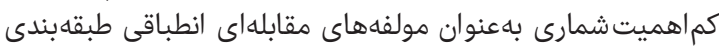

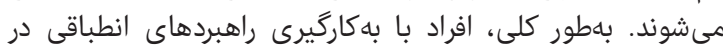

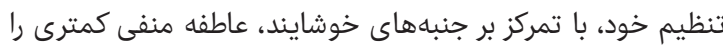

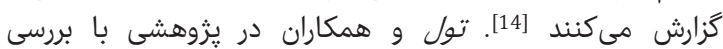

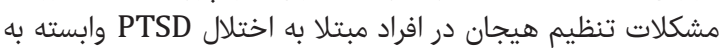

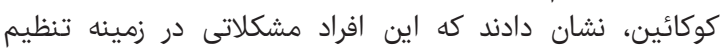

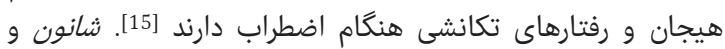

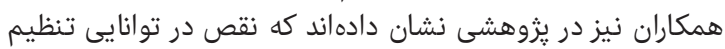

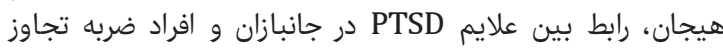

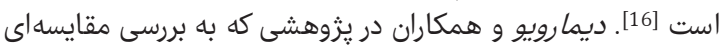

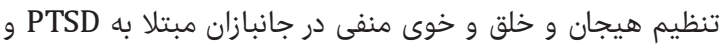
دوره •l، شماره ا، زمستان عهس|

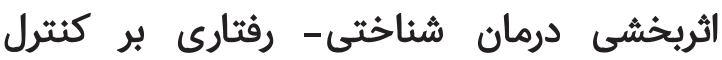 هيجان و افكار خودكشى در جانبازان مبتلا بهان بهان \\ PTSD}

MA "شكوفه رمضانى

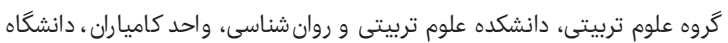

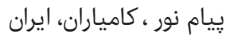

MA نسيم محمدى كامياران ايران

كروه علوم تربيتى، دانشكده علوم تربيتى و روان شناسى، دانشكاه محقق اردبيلى،

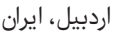

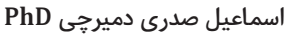

كروه علوم تربيتى، دانشكده علوم تربيتى و روان شناسى، دانشكاه محقق اردبيلى،

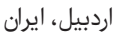

سيف اله رحمانى ترونى

كَروه علوم تربيتى، دانشكده علوم تربيتى و روان شناسى، دانشكاه فرهنكَيان

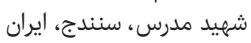

קكيده

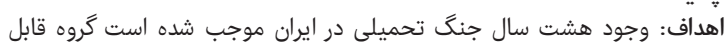

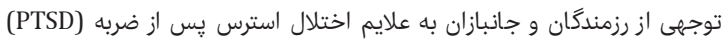

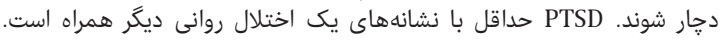

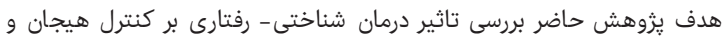

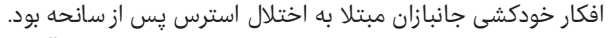

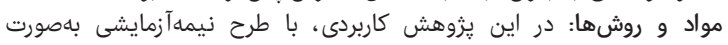

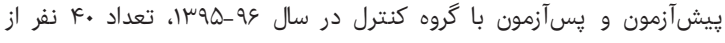

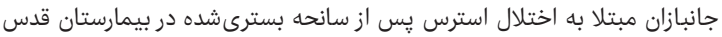

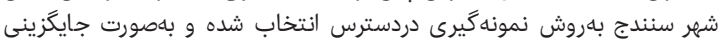

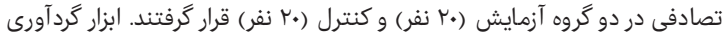

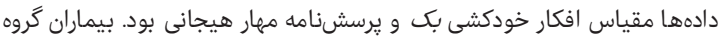

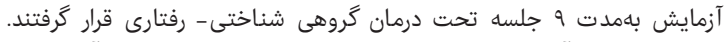

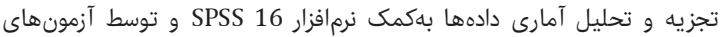

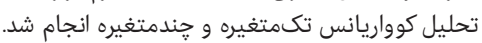

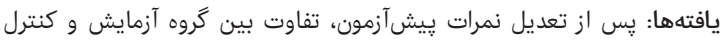

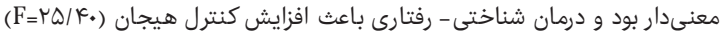

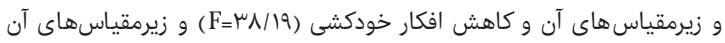

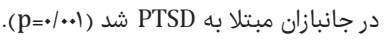

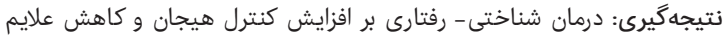

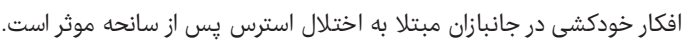

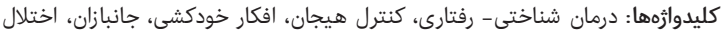

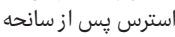

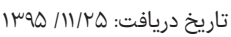

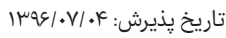

lavinramezani@yahoo.com : نويسنده مسئولثر:"

مقدمه

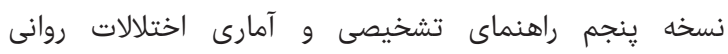

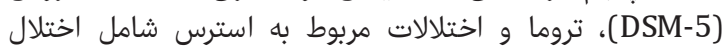

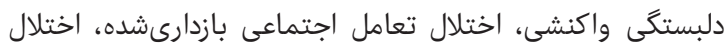

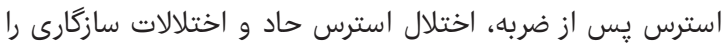

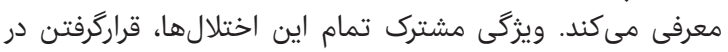

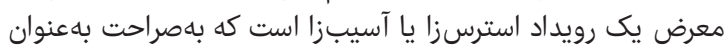

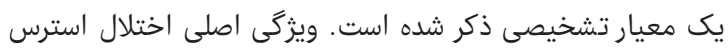

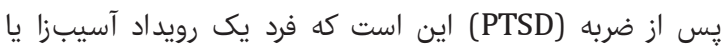

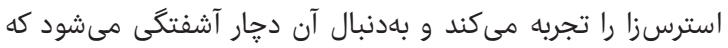

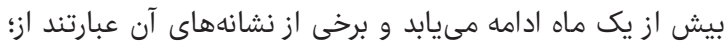

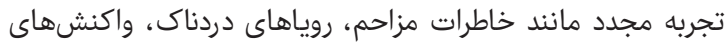




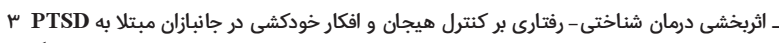

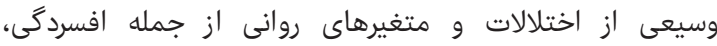

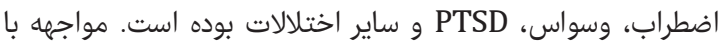

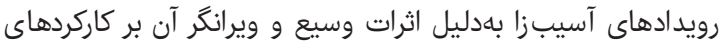

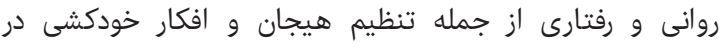

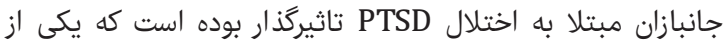

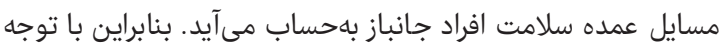

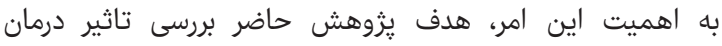

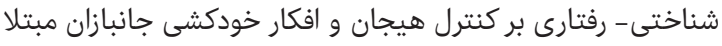

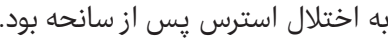

\section{مواد و روشها}

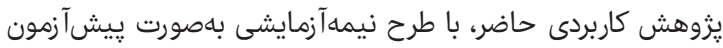

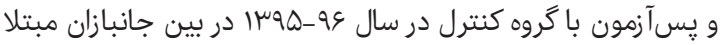

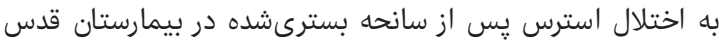

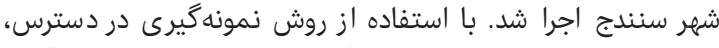

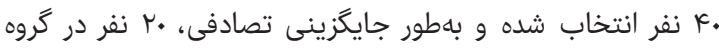

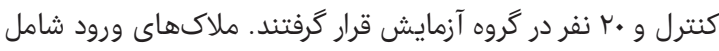

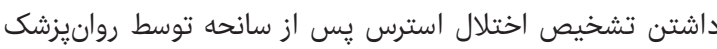

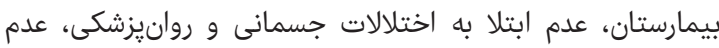

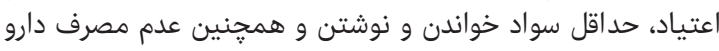

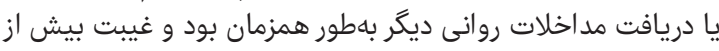

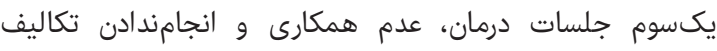

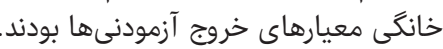

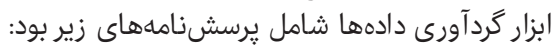

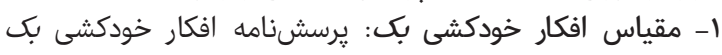

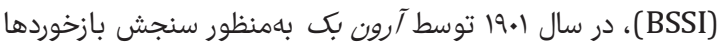

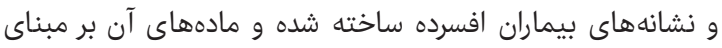

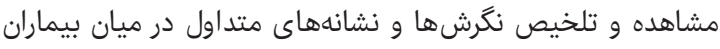

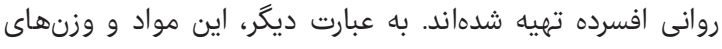

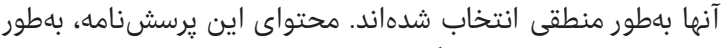

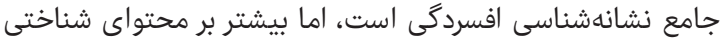

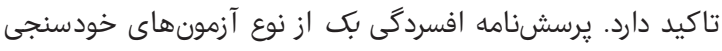

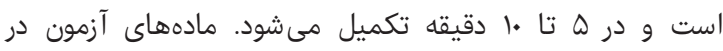

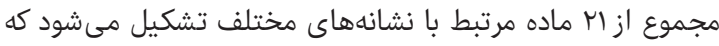

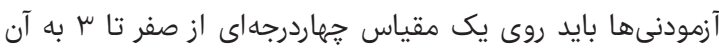

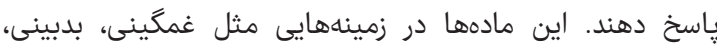

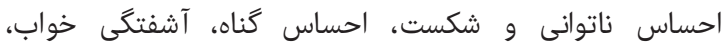

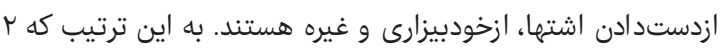

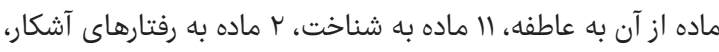

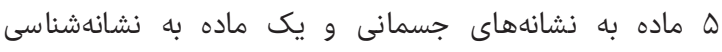

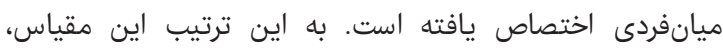

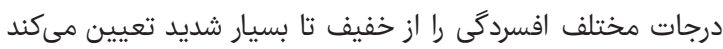

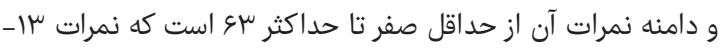

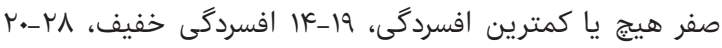

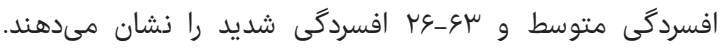

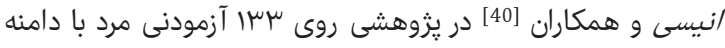

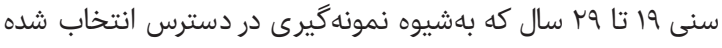

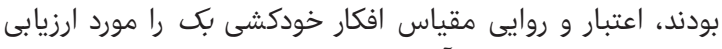

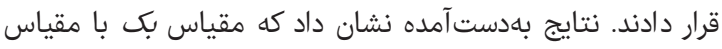

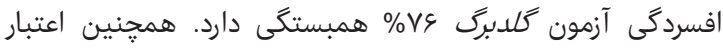

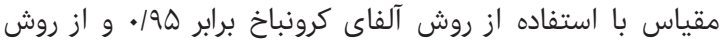

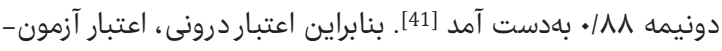

جانبازان بدون اختلال PTSD يرداخت، نشان داد كه جانبازان مبتلا

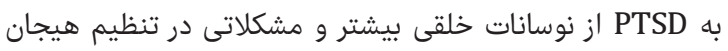

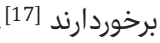

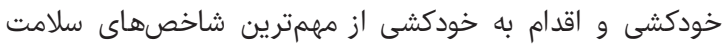

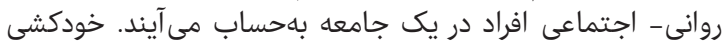

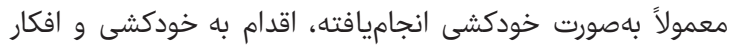

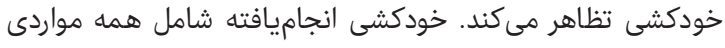

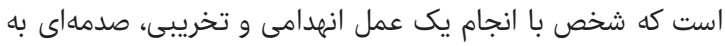

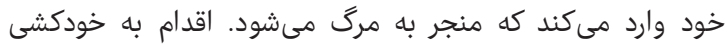

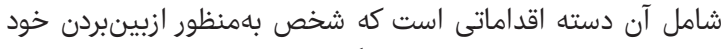

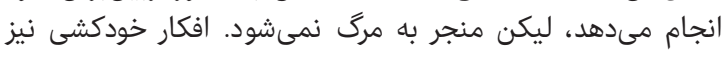

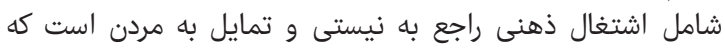

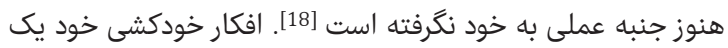

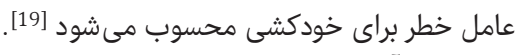

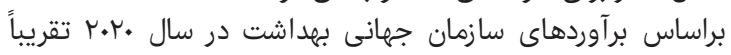

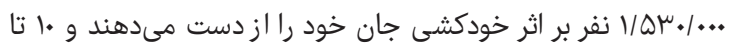

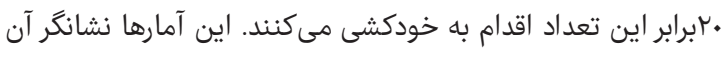

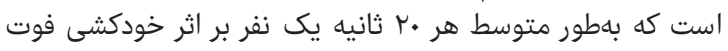

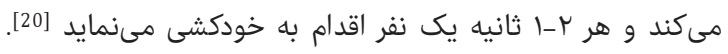

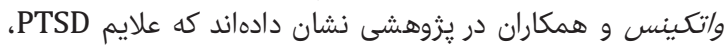

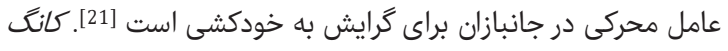

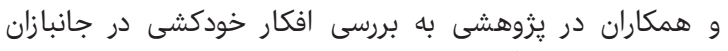

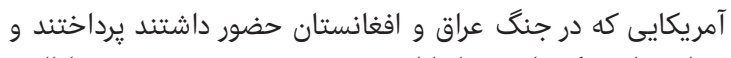

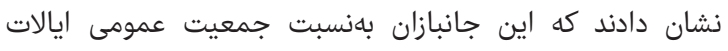

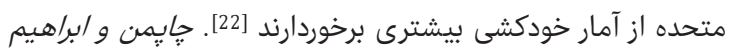

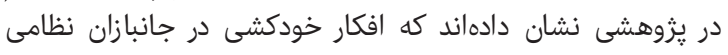

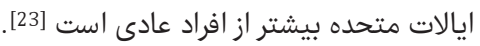

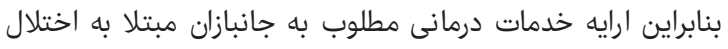

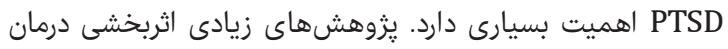

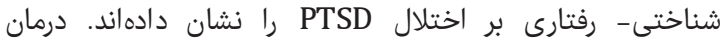

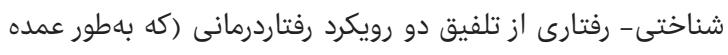

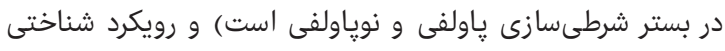

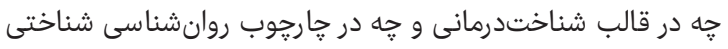

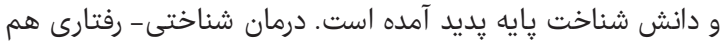

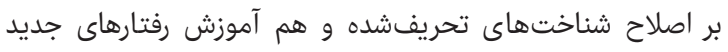

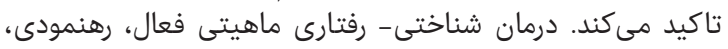

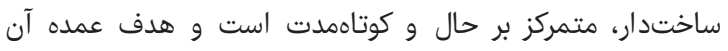

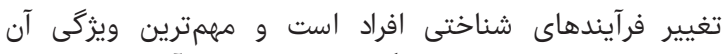

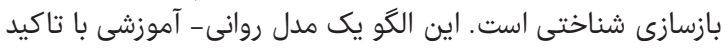

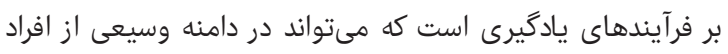

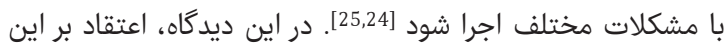

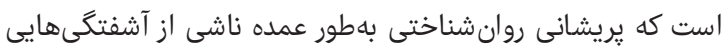

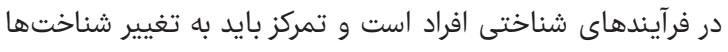

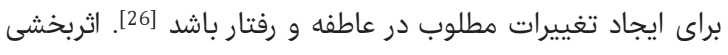

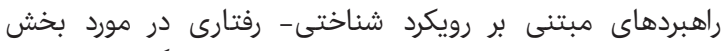

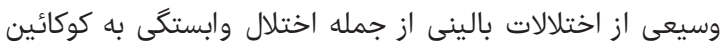

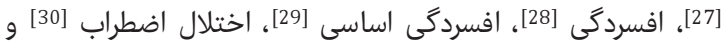

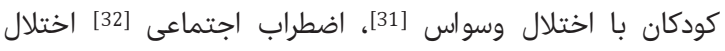

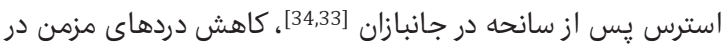

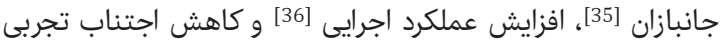
[39,38,37] تاييد شده است. شواهد تجربى حاكى از اثربخشى درمان شناختى- رفتارى در طيف 
بيماران گروه آزمايش بهمدت 9 جلسه •ودقيقهاى، بهصورت يك ترون

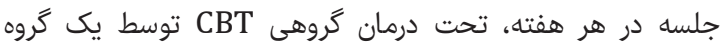

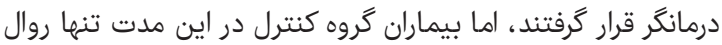

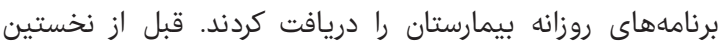

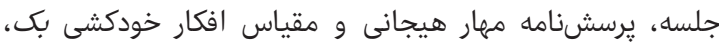

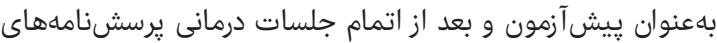

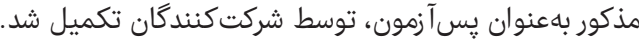

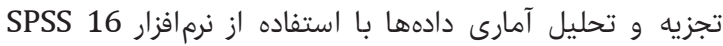

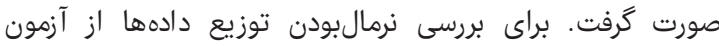

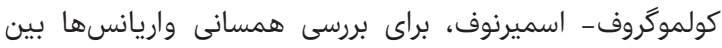

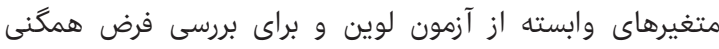

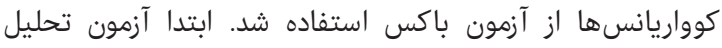

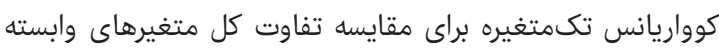

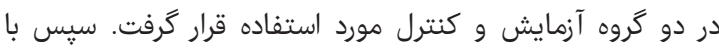

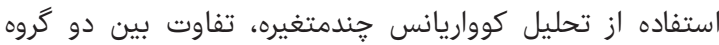

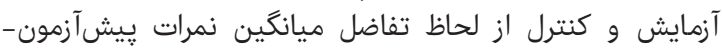

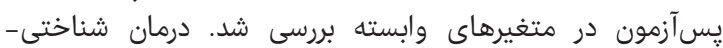

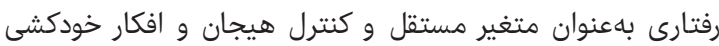
بهنوان متغير وابسته در نظر گرفته شدند.

يافتهها

ميانگين سنى در گروه آزمايش و كنترل به ترتيب سال و و ه D  每

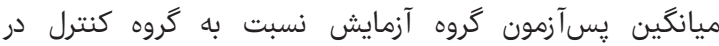

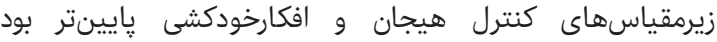

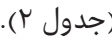

جدول r) مقايسه ميانكَين آمارى نمرات مولفههاى كنترل هيجان و و افكار

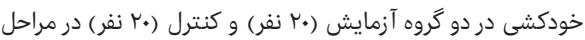

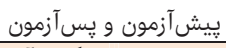

\begin{tabular}{|c|c|c|}
\hline گروه كنترل & كروه آزمايش & متغيرها \\
\hline$r \cdot / \mu s \pm r / \mu r$ & $|\Lambda / \Delta \cdot \pm r| \Lambda \mid$ & بيش بازداري هيجانى \\
\hline$|9 / \varepsilon \Delta \pm r / \&|$ & $r \mu / \mu \Delta \pm r / \Delta K$ & يس" آزمون \\
\hline$r r / I Y \pm \mu / V I$ & $r \cdot /{ }^{\mu} \subseteq \pm \Psi / \Delta V$ & مِيشار يَرخون \\
\hline$r \cdot / r \mu \pm r / \wedge \Delta$ & $r F / \Lambda \Delta \pm Y / G Y$ & يس" آزمون \\
\hline$r \cdot \mid \cdot \psi_{ \pm} \Psi / \varepsilon V$ & 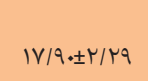 & نشيش رَّار فكرى \\
\hline$Y / / \cdot I \pm Y / \Delta F$ & $r r / \& \Lambda \pm r / V I$ & يسآزمون \\
\hline$r . / q r \pm r / 98$ & $r \cdot / r A \pm r / V K$ & يمهار خوش آزمون \\
\hline$r / \Delta V \pm r / I r$ & $r F / F \mu_{ \pm}+r / F \Lambda$ & يسآزمون \\
\hline 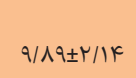 & $1 \cdot / r \pm r / r \Lambda$ & تِيشايل به مرك \\
\hline$q / \Lambda \vee \pm \mu q / r$ & $V / \Delta S \pm I / \Delta \Lambda$ & يس" آزمون \\
\hline$|\varepsilon| \Delta \mu_{ \pm} \Psi / \cdot 1$ & $\mid N / K K \pm F / K \Lambda$ & 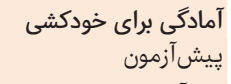 \\
\hline $1 \Delta / ৭ \Delta \pm \mu / \cdot 1$ & $|N / \varsigma 9 \pm \Psi / T|$ & يس آزمون \\
\hline $1 . / 11 \pm r / 11$ & $9 / 4 \wedge \pm r / q \Lambda$ & بيش آزمون خودكشى \\
\hline$q / V \Lambda \pm r / \Delta r$ & $\Delta / s q \pm r / / K$ & يس آزمون \\
\hline
\end{tabular}

بازآزمون و روايى همانى همزاران اين مقياس مورد قبول است. سئوالات

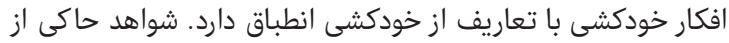

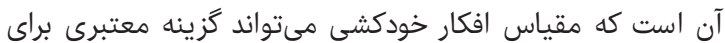

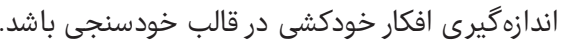

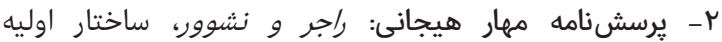

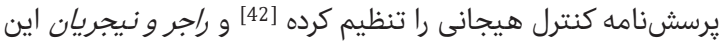

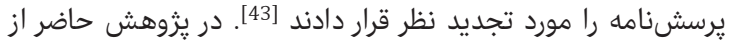

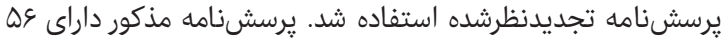

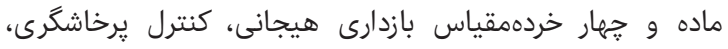

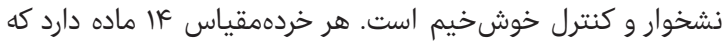

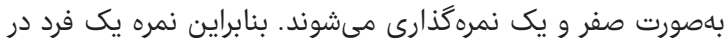

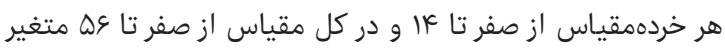

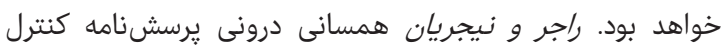

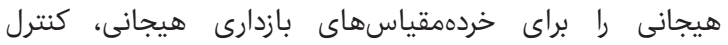

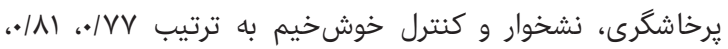

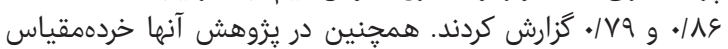

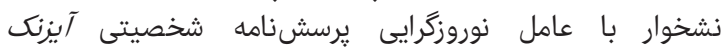

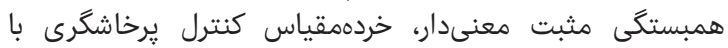

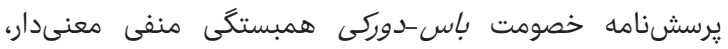

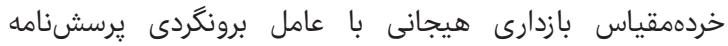

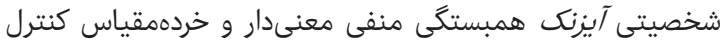

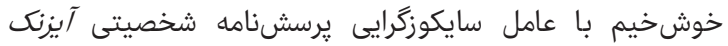

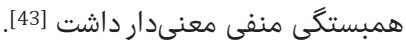

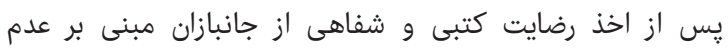

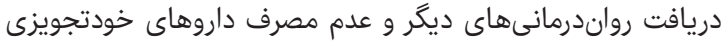

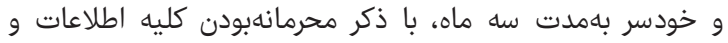

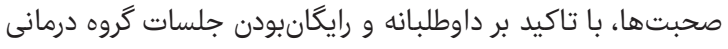

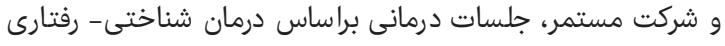
هاوتون و همكاران [38] اجرا شد (جدول (CBT)

جدول () خلاصه جلسات گروهدرمانى شناختى - رفتارى

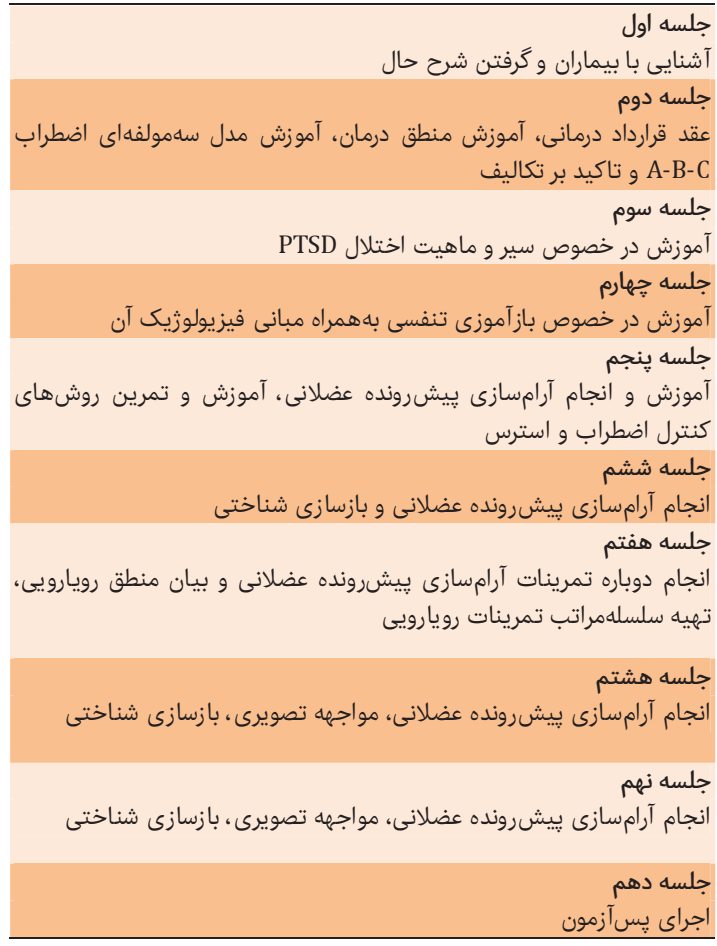




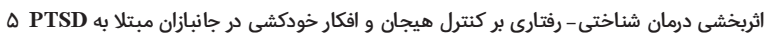

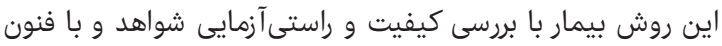

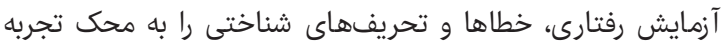

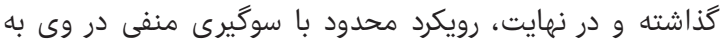

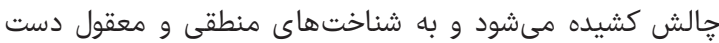
مىيابد [38].

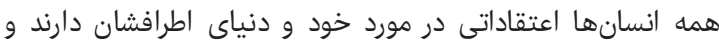

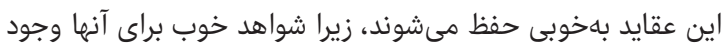

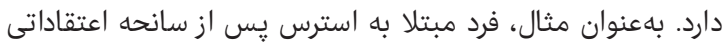

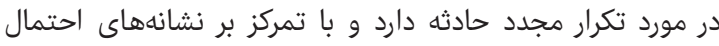

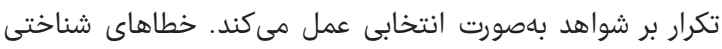

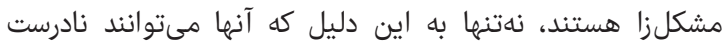

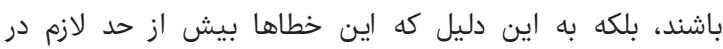

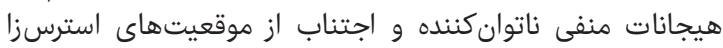

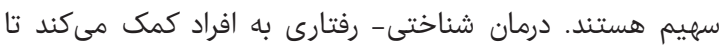

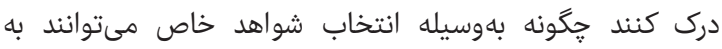

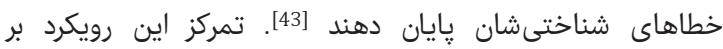

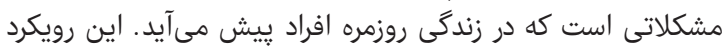

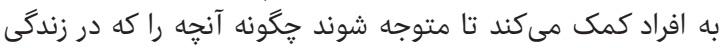

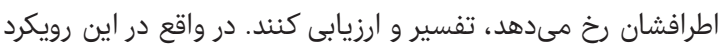

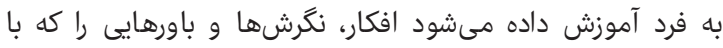

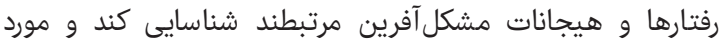

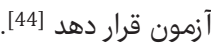

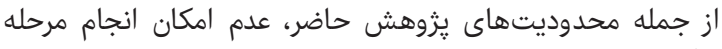

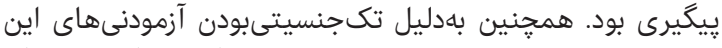

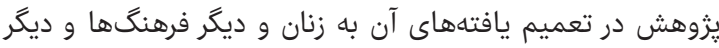

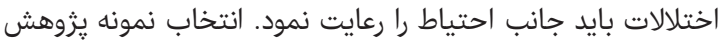

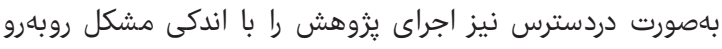

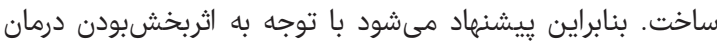

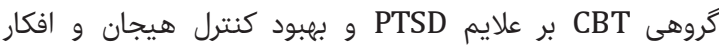

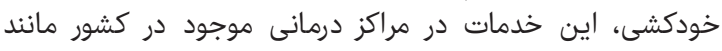

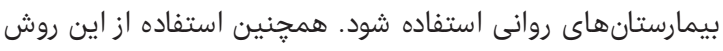

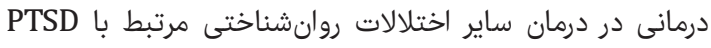
ي يشنهاد مى شود.

\section{نتيجه گيرى}

درمان شناختى- رفتارى بر افزايش كنترل هيجان و كاهش إنش علايم

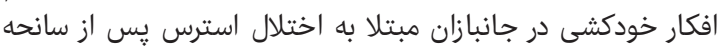

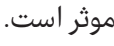

تشكر و قدردانى: از كليه جانبازان و دوستانى كه ما را در اجراى اين

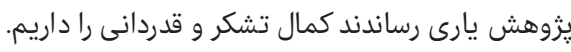

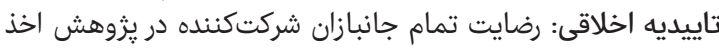

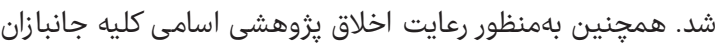

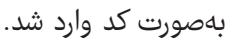

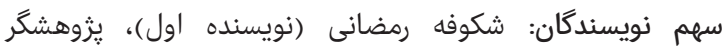

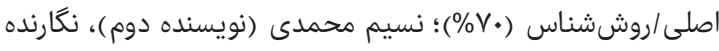

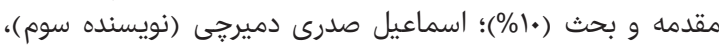

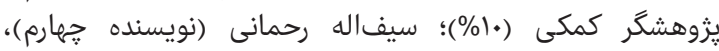

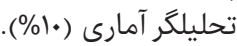

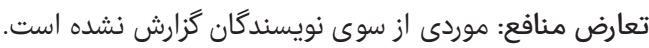

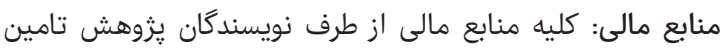

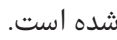

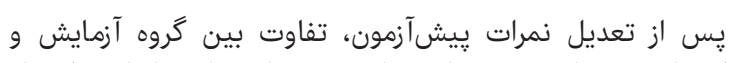

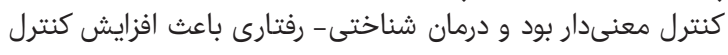

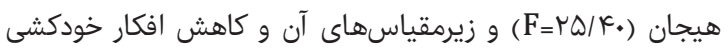
(F=r/N/19)

بحث برهث حاضر با هدف بررسى درمان شناختى ـ رفتارى بر كنترل

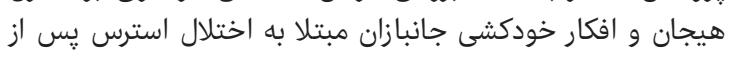

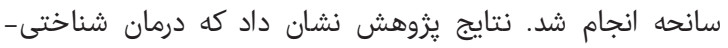

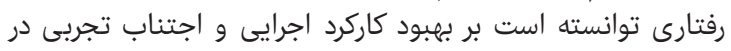

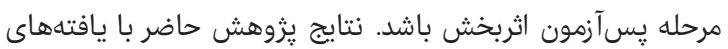

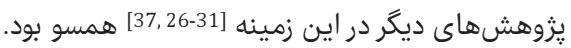

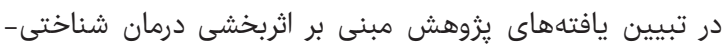

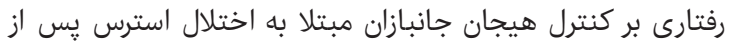

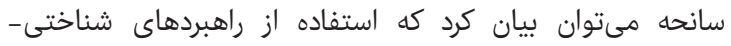

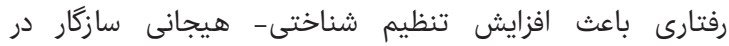

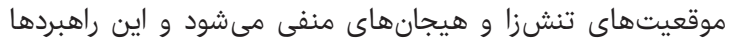

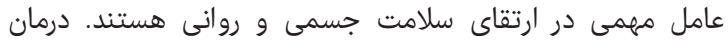

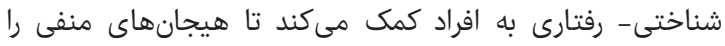

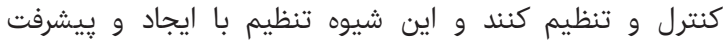

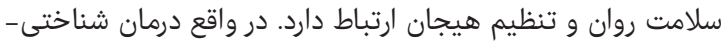

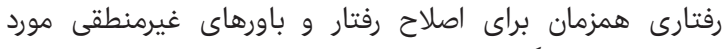

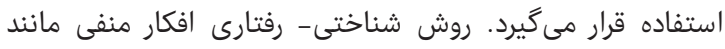

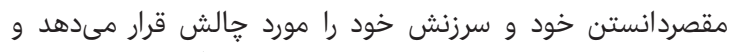

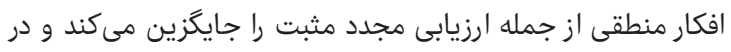

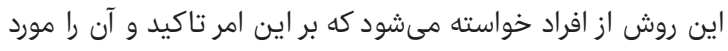

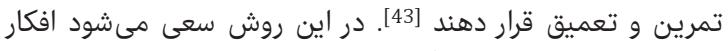

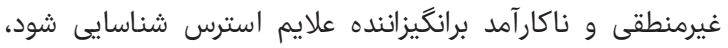

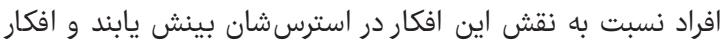

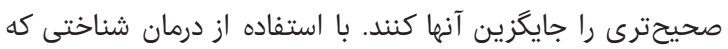

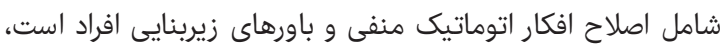

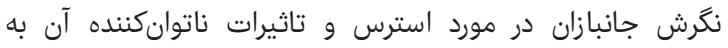

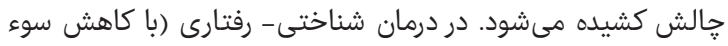

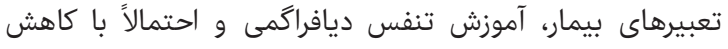

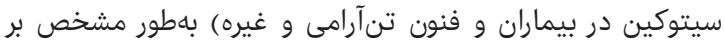

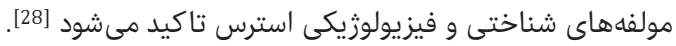

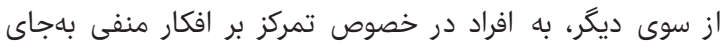

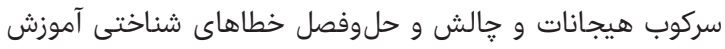

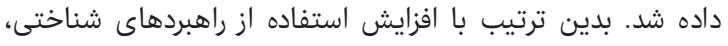

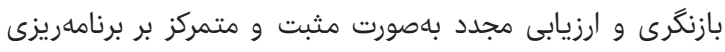

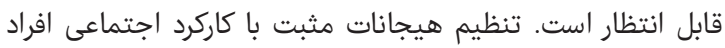

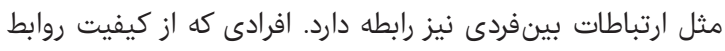

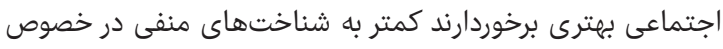

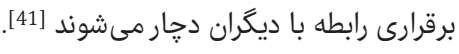

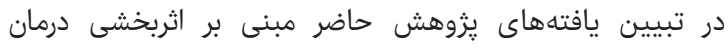

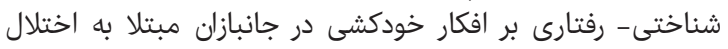

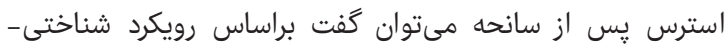

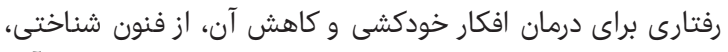

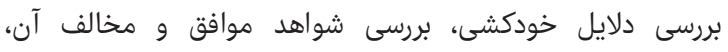

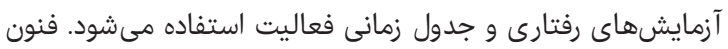

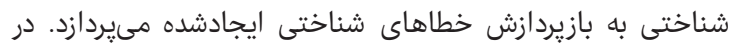

Volume 10, Issue 1, Winter 2018 
18- Fortinash KM, Holoday Worret PA. Psychiatric mental health nursing. $5^{\text {th }}$ edition. Maryland Heights: Mosby; 2011.

19- Groleger U, Tomori M, Kocmur M. Suicidal ideation in adolescence--an indicator of actual risk?. Isr J Psychiatry Relat Sci. 2003;40(3):202-8.

20- Bertolote JM, Fleischmann A. A global perspective in the epidemiology of suicide. Sociol. 2002;7(2):6-8.

21- Watkins LE, Sipped LM, Pietrzak RH, Hoff R, Harpaz Rotem I. Co-occurring aggression and suicide attempt among veterans entering residential treatment for PTSD: The role of PTSD symptom clusters and alcohol misuse. Psychiatr Res. 2017;87:8-14.

22- Kang HK, Bullman TA, Smolenski DJ, Skoop NA, Gahm GA, Reger MA. Suicide risk among 1.3 Million veterans who were on active duty during the Iraq and Afghanistan wars. Ann Epidemiol. 2015;25(2):96-100.

23- Chapman L, Ibrahim H. Identification and management of suicide risk in U.S. military veterans. Tex Med. 2015;111(2):56-60.

24- LaMontagne LL, Hepworth JT, Cohen F, Salisbury MH. Cognitive-behavioral intervention effects on adolescents anxiety and pain following spinal fusion surgery. Nurs Res. 2003;52(3):183-90.

25- Beck AT. The current state of cognitive therapy: A 40-year retrospective. Arch Gen Psychiatry. 2005;62(9):953-9.

26- Rosier JP, Elliott R, Sahakian BJ. Cognitive mechanisms of treatment in depression. Neuron Psycho Rev. 2012;37(1):117-36.

27- Decker SE, Kiluk BD, Frankfurter T, Babuscio T, Niche $\mathrm{C}$, Carroll KM. Just showing up is not enough: Homework adherence and outcome in cognitive-behavioral therapy for cocaine dependence. J Consult Clin Psychol. 2016;84(10):907-12.

28- Johnson TJ, Friborg O. The effects of cognitive behavioral therapy as an anti-depressive treatment is falling: A meta-analysis. Psych Bulletin. 2015; 141(4):747-68.

29- Koenig H, Pearce M, Nelson B, Shaw S, Robins C Dasher N, et al. Religious vs. conventional cognitive behavioral therapy for major depression in persons with chronic medical illness: A pilot randomized trial. J Nerv Ment Dis. 2015;203(4):243-51.

30- Davies CD, Niles AN, Pittig A, Arch JJ, Craske MG Physiological and behavioral indices of emotion deregulation as predictors of outcome from cognitive behavioral therapy and acceptance and commitment therapy for anxiety. J behav Ther Exp Psychiatry. 2015:46:35-43.

31- Öst LG, Riise EN, Wergeland GJ, Hansen B, Kvale G. Cognitive behavioral and pharmacological treatments of OCD in children: A systematic review and meta-analysis. J Anxiety Disord. 2016;43:58-69.

32- Gregory B, Peters L. Changes in the self during cognitive behavioral therapy for social anxiety disorder: A systematic review. Cline Psycho Rev. 2016;52:1-18.

33- Lumberton CM, Madison JF, Bellshill AJ. A case study of individually delivered mindfulness-based cognitive behavioral therapy for severe health anxiety. Cognit behav Pract. 2017;24(4):484-95.

34- Otis JD, Keane TM, Kerns RD, Monson C, Scold E. The development of an integrated treatment for veterans with comorbid chronic pain and posttraumatic stress disorder. Pain Med. 2009;10(7):1300-11.

35- Miyahira SD, Folen RA, Hoffman HG, Garcia Palacios A, Schaper KM. Effectiveness of brief VR treatment for

1- American Psychiatric Association. Diagnostic and statistical manual of mental disorders (DSM). Arlington: American Psychiatric Association; 2013. pp. 175-272. 2- Chossegros L, Hours M, Charnay P, Bernard M, Fort E, Buisson D, et al. Predictive factors of chronic posttraumatic stress disorder 6 months after a road traffic accident. Acid Analysis Prev. 2011;4(43):471-7.

3- Alipoor M, Lorestani F. I and post-traumatic stress disorder. Tehran: Janbazan Medical and Engineering Research Center Publication; 2005. [Persian]

4- Ahmadi Kh, Zarei A, Arabnia AR. The study of effective factors on fmarital aladjusment among veterans with PTSD. J Mil Med. 2006;8(3):165-74. [Persian]

5- Shafiee Kamalabadi M, Bigdeli I, Alavi K, Kianersi F. Prevalence of Post-Traumatic stress disorder and comorbid personality disorders in the groups veterans Tehran City. J Clan Psycol. 2014;6(1):75-65. [Persian] 6- Mohaghegh Motlagh J, Momtazi S, Musavi Nasab N, Arab A, Saburi E, Saburi A. Post-traumatic stress disorder in male chemical injured war veterans compared to nonchemical war veterans. J Mashhad Univ Med Sci. 2013;56(6):362-8. [Persian]

7- Horesh D, Lowe SR, Galena S, Aiello AE, Udine M, Koenan KC. An in-depth look into PTSD-depression comorbidity: A longitudinal study of chronically-exposed Detroit residents. J Affect Disord. 2017;208:653-61.

8- Tay AK, Rees S, Chan J, Kareth M, Silove D. Examining the broader psychosocial effects of mass conflict on PTSD symptoms and functional impairment amongst West Papuan refugees resettled in Papua New Guinea (PNG). Sod Sic Med. 2015;132:70-8.

9- McDevitt Murphy ME, Luciano MT, Tripp JC, Eddinger JE. Drinking motives and PTSD-related alcohol expectancies among combat veterans . Addict Behav. 2017;64:217-22.

10- Bowen S, De Boer D, Bergman AL. The role of mindfulness as approach-based coping in the PTSDsubstance abuse cycle. Addict Behav. 2017;64:212-6.

11- Simons JS, Simons RM, O'Brien C, Stoltenberg SF, Keith JA, Hudson JA. PTSD, alcohol dependence, and conduct problems: Distinct pathways via liability and disinhibit ion. Addict Behav. 2017;64:185-93.

12- Brown WJ, Wilkerson AK, Milbank ME, Turk PC, Huda Tw, Cortes' BL, et al. An examination of sleep quality in veterans with a dual diagnosis of PTSD and severe mental illness. Psychiatry Res. 2017;247:15-20.

13- Poindexter E, Mitchell MS, Jahn DR, Smith PN, Hirsch JK, Cukrowic K. PTSD symptoms and suicide ideation: Testing the conditional indirect effects of thwarted interpersonal needs and using substances to cope. Person Indiv Difference. 2015;77:167-72.

14- Moriarty N, Stough C, Tidmarsh P, Eqer D, Dennison S. Deficit in emotional intelligence underlying adolescent set offending. J Adolesc. 2001;24(6):743-51.

15- Tull MT, Gratz KL, McDermott MJ, Bordieri MJ, Daughters SB, Lejuez CW. The role of emotion regulation difficulties in the relation between ptsd symptoms and the learned association between Trauma-related and Cocaine cues. Subst Use Misuse. 2016;51(10):1318-29. 16- Miles SR, Thompson KE, Stanley MA, Kent TA. Singlesession emotion regulation skills training to reduce aggression in combat veterans: A clinical innovation case study. Psychol Serv. 2016;13(2):170-7.

17- DiMauro J, Renshaw KD, Kashdan TB. Beliefs in negative mood regulation and daily negative affect in PTSD. Personal Endiv Difference. 2016;95:34-6. 
اثربخشى درمان شناختى - رفتارى بر كنترل هيجان و افكار خودكشى در جانبازان مبتلا به P PTSD

40-Anise J, Fathi Asthenia A, Salimi S, Ahamada Node Kh. Assessing the reliability and validity of the beck suicidal thoughts scale (BSSI) in Soldiers. J Mil Med. 2005;7(1):33-7.

41- Ravinia P. The relationship between emotional expression styles with general health of Students [Dissertation]. Tehran: Terabit Moderns University; 2001. [Persian]

42- Roger D, Nesshoever W. The construction and preliminary validation of a scale for measuring emotional control. Person Indivi Diff. 1987;8(4):527-34. 43- Roger D, Nigerian B. The construction and validation of a new scale for measuring emotion control. Person Indivd Diff. 1989;10(8):845-53.

44- Bagherinia $H$, Yamani $M$, Javadielmi L, Nooradi T. Comparison of the efficacy of cognitive behavior therapy and mindfulness-based therapy in improving cognitive emotion regulation in major depressive disorder. Armaghane Danesh. 2015;20(3):210-19. [Persian]

PTSD in war-fighters: A case study. Stud Health Technol Inform. 2010;154:214-9.

36- Thompson DG, Kessler SR, Sondheim K, Mehta KM, Thompson LW, Marquette RM, et al. FMRI activation during executive function predicts response to cognitive behavioral therapy in older, depressed adults. Am J Geriatr Psychiatry. 2015;23(1):13-22.

37- Glister AT, Klotche J, Gerlach AL, Hamm A, Strole A, Gauggel S, et al. Timing matters: change depends on the stage of treatment in cognitive behavioral therapy for panic disorder with agoraphobia. J Consult Clin Psychol. 2014;82(1):141-53.

38- Houghton K, Crack y, Salcomo C, Pal C, David M. Cognitive behavioral therapy [Volume I]. Ghasemzadeh H, translator. Tehran: Arjomand; 2003.

39- Espoo EP, Gorlic A, Castriottta N. Changes in threatrelated cognitions and experiential avoidance in groupbased transdiagnostic CBT for anxiety disorders. 2017;46(2):65-71. 